\title{
Dissociative recombination and its impact on the line profile of the hydrogen Balmer series
}

\author{
R Friedl ${ }^{1}$, D Rauner ${ }^{1}$, A Heiler ${ }^{1,2}$ and U Fantz ${ }^{1,2}$ \\ ${ }^{1}$ AG Experimentelle Plasmaphysik, Universität Augsburg, 86135 Augsburg \\ 2 Max-Planck-Institut für Plasmaphysik, Boltzmannstr. 2, 85748 Garching \\ E-mail: roland.friedl@physik.uni-augsburg.de
}

\begin{abstract}
In low pressure low temperature plasmas $\left(\mathrm{p} \sim \mathrm{Pa}, T_{\mathrm{e}} \sim \mathrm{eV}\right)$ the upper levels of the Balmer series of the hydrogen atom are populated by several excitation channels. While electron impact excitation is dominant for so-called ionizing plasmas $\left(T_{\mathrm{e}} \rightarrow 10 \mathrm{eV}\right)$, dissociative recombination (DR) via the molecular ion $\mathrm{H}_{2}^{+}$becomes dominant in so-called recombining plasmas $\left(T_{\mathrm{e}} \rightarrow \lesssim 1 \mathrm{eV}\right)$. In the intermediate range, several other channels may be relevant as well. The expansion region of an ECR discharge, which represents such a plasma, is analyzed regarding the composition of the excitation processes as well as the line profiles of the Balmer series (for $\mathrm{H}_{\alpha}$ to $\mathrm{H}_{\delta}$ ). Significantly broadened line profiles compared to Doppler broadening of thermal hydrogen atoms are revealed. The resulting line profiles resemble two Gaussian components with distinct FWHM (narrow component close to apparatus profile: $\approx 25-30 \mathrm{pm}$; broad component: $\approx 50-100 \mathrm{pm}$ ), where the broad part contributes to $10-40 \%$ to the emissivity, depending on the individual Balmer line and the plasma conditions. Collisional radiative modeling was applied in order to determine the contributions of the different excitation channels. It turned out that for each of the lines of the Balmer series, population via DR contributes to the total excitation with a similar share as the share of the broad component of the line profile. Hence, it is concluded that DR is responsible for the broad component in the Balmer line profiles. The implication of this analysis on the spectroscopic determination of the atomic hydrogen density using a simple line ratio method $\left(\mathrm{H}_{\gamma} /\right.$ Fulcher $)$ is illustrated.
\end{abstract}

Keywords: low temperature plasma, hydrogen, Balmer series, collisional radiative modeling, line profile, dissociative recombination, atomic density

Submitted to: Plasma Sources Sci. Technol. in August 2019, v1.1, revised 28 October 2019 


\section{Introduction}

The Balmer series of the hydrogen atom (optical transition $n \geq 3 \rightarrow n^{\prime}=2$ ) is one of the most common observables of hydrogen discharges since it lies in the visible spectral range $(\approx 370-656 \mathrm{~nm})$ and is thus easily detectable. The entirety of its emission lines is capable of giving a non-invasive insight into the occurring plasma processes, since the upper states of the Balmer series can be populated by several different channels involving all the hydrogenic species (see [1-5] for instance).

The relevance of a specific channel depends on the particular plasma regime and can be determined by reconstruction of measurements using collisional radiative (CR) models. For low pressure low temperature (LPLT) hydrogen plasmas it is known that electron impact excitation (EE) from the hydrogen atom, dissociative excitation (DE) from the hydrogen molecule and dissociative recombination (DR) from the molecular ion $\mathrm{H}_{2}^{+}$are the dominant population processes for the Balmer series $[4,5]$. The detailed analysis leading to this selection is recapitulated in section 2. Further subdivision of the prevailing excitation processes can be done by means of the electron temperature $T_{\mathrm{e}}$ : in plasmas with high $T_{\mathrm{e}}$, which are commonly called "ionizing plasmas", EE and $\mathrm{DE}$ via $\mathrm{H}$ and $\mathrm{H}_{2}$, respectively, will dominate the total excitation rate for Balmer emission. On the other hand, for the low electron temperatures of "recombining plasmas", dissociative recombination via $\mathrm{H}_{2}^{+}$can be the dominant channel.

The combination of measuring the Balmer series and CR modeling can moreover give access to the densities and/or temperatures of the respective educt species - atoms, molecules, ions and electrons - , which is widely used as diagnostic tool: optical emission spectroscopy (OES). As an example, the line ratio of Balmer lines to the molecular Fulcher transition is frequently utilized for an assessment of the atomto-molecule density ratio and thus of the atomic hydrogen density [6-11]. Typically, only the EE channel is considered in the evaluation procedure and, expectably, severe discrepancies may occur when further processes like DE or DR contribute to the measured emission, which will be shown later on.

Apart from the absolute emissivity of a Balmer line, specific population channels can also have an influence on its spectral line profile. For instance, energy release during the excitation process can result in a hot translational energy distribution of the emitting state and consequently to a strongly broadened line profile. This and other effects related to the line profile are discussed in section 3. Consequently, besides CR modeling, the line profiles of the Balmer series are another tool to gain access to the occurring plasma processes in hydrogen discharges [12-27].

This paper describes the analysis of a hydrogen discharge in a region of plasma expansion, i. e. in a situation where all the three channels (EE, DE, DR) play a role. The emissivities of the first four Balmer lines $\mathrm{H}_{\alpha}$ through $\mathrm{H}_{\delta}$ are evaluated by collisional radiative modeling and the respective line profiles are thoroughly analyzed. For the latter it will be shown, that two distinct components (a "hot" and a "cold" one) can be identified and the respective widths as well as the area shares of the two components are correlated to the occurring plasma processes EE and DR. The influence of neglecting the DR channel for the evaluation of plasma parameters (here the atomic density) via OES will be highlighted.

\section{Exciting the Balmer series in low temperature plasmas}

Figure 1 (a) shows exemplarily the population coefficients for excitation of the level $n=5$ by several electron collision processes: electron impact excitation of hydrogen atoms $(\mathrm{H}+\mathrm{e})$, dissociative excitation from hydrogen molecules $\left(\mathrm{H}_{2}+\mathrm{e}\right)$ as well as the different recombination channels from the ionic species $\left(\mathrm{H}_{x}^{+}+\mathrm{e}\right.$, for $\left.x=1,2,3\right)$. The coefficients are calculated with the collisional radiative $(\mathrm{CR})$ model Yacora $\mathrm{H}$ [5] for an electron density $n_{\mathrm{e}}$ of $3 \times 10^{16} \mathrm{~m}^{-3}$. Redistribution processes within the hydrogen atomic states are included for each of the population channels. This means that, for instance, direct population by dissociative excitation from hydrogen molecules is followed by optical as well as electron-collision-induced transitions among the hydrogen states. The depicted population coefficient " $\mathrm{H}_{2}+\mathrm{e}$ " represents the one for equilibrium population of $n=5$ due to all the included and redistributed dissociative excitation reactions (see [5] for more detail). In part (b) of figure 1 the corresponding contributions to the effective emission rate coefficient for the $\mathrm{H}_{\gamma}$ line in equilibrium are shown, assuming a density distribution of the hydrogen species which is representative for LPLT hydrogen plasmas.

While dissociative recombination via the $\mathrm{H}_{3}^{+}$ion predominantly populates the $n=2$ state, recombination from the proton $\mathrm{H}^{+}$(which includes three-body as well as radiative recombination) mainly leads to high- $n$ states and furthermore requires high plasma densities and low electron temperatures to be effective [1]. Consequently, even after redistribution, those processes give only a negligible contribution to the effective emission rate coefficient of the $\mathrm{H}_{\gamma}$ line, as can be seen in figure 1 (b). Moreover, they are in general only of minor relevance for a discussion on all the commonly observed Balmer lines in LPLT hydrogen plasmas. Hence, for electron densities $n_{\mathrm{e}}$ in the range 
(a)

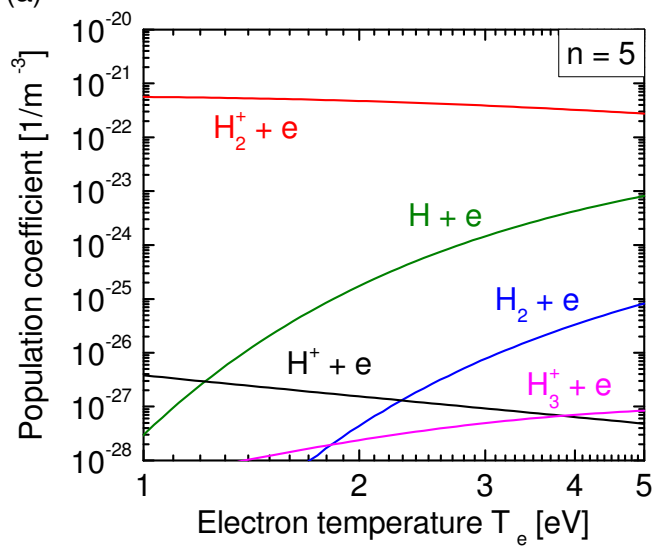

(b)

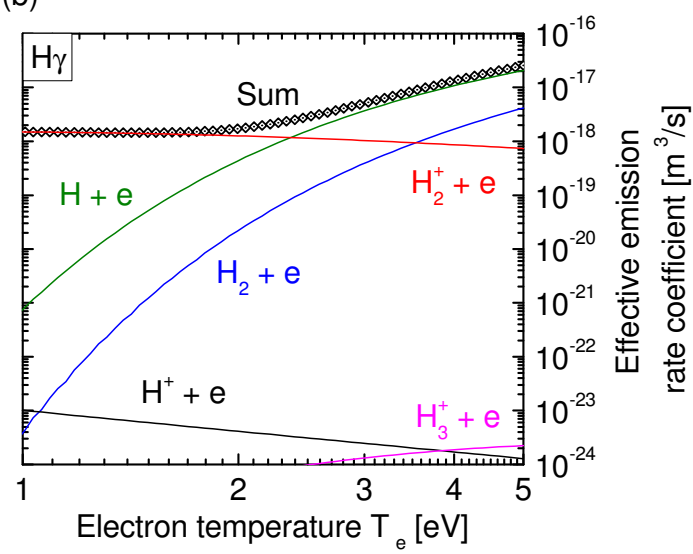

Figure 1. (a) Population coefficients for excitation of the state $n=5$ of the hydrogen atom, calculated with the collisional radiative model Yacora $\mathrm{H}$ [5] for $n_{\mathrm{e}}=3 \times 10^{16} \mathrm{~m}^{-3}$. (b) Effective emission rate coefficient ("Sum") for the $\mathrm{H}_{\gamma}$ line $(n=5 \rightarrow 2$, $\lambda=434.05 \mathrm{~nm}$ ) together with the respective contributions from the channels shown in part (a). The following species densities were used: $n_{\mathrm{H}_{2}}=2 \times 10^{20} \mathrm{~m}^{-3}, n_{\mathrm{H}}=1 \times 10^{19} \mathrm{~m}^{-3}$ and $n_{\mathrm{H}^{+}}=n_{\mathrm{H}_{2}^{+}}=n_{\mathrm{H}_{3}^{+}}=1 / 3 n_{\mathrm{e}}=1 \times 10^{16} \mathrm{~m}^{-3}$.

of $10^{15}-10^{18} \mathrm{~m}^{-3}$ and electron temperatures $T_{\mathrm{e}}$ of $1-$ $10 \mathrm{eV}$, the most relevant channels resulting in Balmer emission are [5] electron impact excitation (EE) from the hydrogen atom, dissociative excitation (DE) from the hydrogen molecule and dissociative recombination (DR) from the molecular ion $\mathrm{H}_{2}^{+}$:

$\mathrm{EE}$ :

$$
\mathrm{H}+\mathrm{e}^{-} \longrightarrow \mathrm{H}(n)+\mathrm{e}^{-},
$$

$\mathrm{DE}:$

$$
\mathrm{H}_{2}+\mathrm{e}^{-} \longrightarrow \mathrm{H}(n)+\mathrm{H}(1)+\mathrm{e}^{-} \text {, }
$$

DR :

$$
\mathrm{H}_{2}^{+}+\mathrm{e}^{-} \longrightarrow \mathrm{H}(n)+\mathrm{H}(1) \text {. }
$$

The written reaction equations (1)-(3) only represent the starting reaction, while for each of the individual channels redistribution processes occur afterwards within the $n$-system. However, the share of these channels to the effective emission rate coefficient in equilibrium still mainly depends on the rate of these initial reactions, and thus on the densities of the involved collision partners $\left(\mathrm{H}, \mathrm{H}_{2}, \mathrm{H}_{2}^{+}\right)$and on the electron temperature. Here, the rate coefficient of the excitation processes from the neutrals $\mathrm{H}$ and $\mathrm{H}_{2}$ show the typical steep dependency from the electron temperature due to the existing threshold energies $(13.1 \mathrm{eV}$ for $\mathrm{EE}, \leq 17 \mathrm{eV}$ for $\mathrm{DE}$ depending on the vibrational excitation of $\left.\mathrm{H}_{2}[1,28]\right)$. In contrast, the threshold for dissociative recombination is negligible [1] and thus, the rate coefficient for this channel is only slightly depending on $T_{\mathrm{e}}$. These dependencies can still be seen in figure 1 (b) for the equilibrium after redistribution. In consequence, in plasmas with high electron temperatures, EE and DE will dominate the total excitation rate, while for low electron temperatures and/or comparatively low atomic densities, dissociative recombination can be the dominant excitation channel for the Balmer seriesł.

$\ddagger$ Obviously, also the other recombinative processes for

\section{Line broadening in low pressure low temperature hydrogen plasmas}

Besides natural line broadening, the line profile of a particular Balmer line $n \rightarrow n^{\prime}$ is determined by the translational energy distribution of the particle ensemble in the upper state $n$ as well as by possible disturbances of the energy of the involved levels $n$ and $n^{\prime}$. For the former effect, the actual process populating the upper state is the determining factor, while for the latter case, effects like Stark or pressure broadening and Zeeman splitting might by relevant.

Stark broadening results from the interaction of the excited hydrogen atom-more precisely of its dipole moment - with the electric field generated by the charged plasma species [29]. It is a well-known mechanism for broadening of Balmer lines [20-27], and its influence depends strongly on the ion or electron density and the respective emission line (increasing with increasing quantum number). However, even for the Balmer line emitting from $n=8$ the FWHM due to Stark broadening for $n_{\mathrm{e}}=10^{18} \mathrm{~m}^{-3}$ is below $10 \mathrm{pm}$ [23, 26]. Hence, for the Balmer lines analyzed in the current LPLT plasmas $\left(n \leq 6, n_{\mathrm{e}}<10^{17} \mathrm{~m}^{-3}\right)$, this mechanism is negligible compared to other effects described below. Collisions of the emitting hydrogen atom with other neutral particles in the plasma is another cause for line broadening. Due to the interaction the effective lifetime of the emitting state is reduced which increases the energy uncertainty of the state and thus, the so-called van-der-Waals broadening of the Balmer emission line may occur [29]. However, due to the

populating the $n=2$ and high- $n$ states via $\mathrm{H}_{3}^{+}$and $\mathrm{H}^{+}$, respectively, gain relevance under such conditions, which is illustrated in [5] for instance. 
low pressure of LPLT plasmas (typically only several $\mathrm{Pa})$ together with the short range of the van-der-Waals force, this mechanism can as well be neglected for the present considerations. The presence of magnetic fields can also result in broadening of the emission lines due to the Zeeman splitting of the fine structure levels (in the weak-field approximation) [29]. However, for typical magnetic field strengths of less than $100 \mathrm{mT}$ this effect can again be seen as negligible [28].

The most important influence on the line profile of emission lines in hydrogen LPLT discharges is thus the translational energy distribution of the emitting state, which is discussed in numerous papers [1219]. If the energy distribution can be described by a Maxwell-Boltzmann distribution, the effect is referred to as Doppler broadening and the resulting line profile is a Gaussian profile whose full width at half maximum (FWHM) depends on the temperature of the emitting state. Consequently, a discussion on the line profile is directly transferred to a discussion of the energy distribution of the state $n$ and thus to its underlying population process. If the upper state $n$ is populated by several processes with specific impacts on its translational energy distribution, the resulting line profile may even be composed of several components, where the composition depends on the relative contribution of the different channels.

In the following, the three main excitation channels for Balmer emission EE, DE and DR are discussed regarding their fundamental influence on the line profile. Only the initial direct processes (1)-(3) are considered due to their presumable dominant influence on the equilibrium translational energy distribution, while possible secondary influences arising from redistribution are neglected here.

EE During an EE process (1) the translational energy distribution of the atom is directly transferred to the excited state due to two reasons: (i) electrons colliding with hydrogen atoms cannot effectively transfer momentum and (ii) the rate for electron collision excitation is only negligibly depending on the translational energy of the hydrogen atom (the relative energy is still defined by the electron energy). Hence, independently of the actual excited state its energy distribution is determined by the energy distribution of the ground state hydrogen atoms. The Doppler line profiles should thus be independent of the specific line (apart from the dependency from the wavelength). The FWHM for an atomic hydrogen temperature of $1000 \mathrm{~K}$ would for instance be $15 \mathrm{pm}$ for $\mathrm{H}_{\alpha}$ down to $9 \mathrm{pm}$ for $\mathrm{H}_{\delta}$.

In LPLT plasmas, atoms are mainly produced by electron impact excitation of ground state hydrogen molecules into the repulsive state $\mathrm{b}^{3} \Sigma_{\mathrm{u}}^{+}$, which leads to immediate dissociation into two ground state hydrogen atoms. The atoms gain kinetic energy by leaving the molecular potential via the anti-bonding curve. This so-called FranckCondon-Energy (FCE) reaches up to more than $2 \mathrm{eV}$ per atom. In equilibrium an atomic energy distribution can be obtained, which exhibits a distinct high-energy tail (see [30] for instance). Thus, broadened line profiles with FWHM of more than $70 \mathrm{pm}$ for $\mathrm{H}_{\alpha}$ (45 pm for $\mathrm{H}_{\delta}$ ) can be achieved. If the energy distribution of the hydrogen atoms can be described by a "cold" and a "hot" part, also the resulting line profile is composed of two components and the share of these components is constant for all emission lines.

DE Dissociation of hydrogen molecules can also directly lead to hydrogen atoms in an excited state via the dissociative excitation channel (2). This process proceeds via highly excited molecular states and thus, energetic excited atoms can be produced by leaving the molecular potentials [14, 28]. As long as the radiative de-excitation rate is higher than the rate for thermalizing collisionswhich is the case for the present investigations, this "hot" ensemble should be observable in emission.

Again, the obtained energy is in the range of some $\mathrm{eV}$, and FWHM of up to more than $100 \mathrm{pm}$ for $\mathrm{H}_{\alpha}$ might be expected [13-15,28]. However, in this case, the final translational energy gained by the atom in the excited state depends on the actual reaction path and the related final state. Hence, different lines are not necessarily broadened equally.

If this population process occurs together with the EE process - which is typically the case - the line profile is composed of several components. In this case, the share of the different components should reflect the share of the population channels to the effective emission rate coefficient. Here, the $\mathrm{DE}$ process is a so-called "bottom-up-process", which means that the states are populated from lower-lying states - the molecular ground state in this case. This results in decreasing population coefficients with increasing quantum number. This is illustrated in figure 2 where the population coefficients for the states $n=3$ through $n=6$ are shown for the DE process in relation to the $\mathrm{EE}$ process (lower part). The decreasing relative population coefficient for increasing quantum number can readily be seen. Regarding the composed line profile, this effect leads to decreasing shares of the DE-related component with increasing quantum number.

DR The DR process (3) proceeds via a doubly excited 


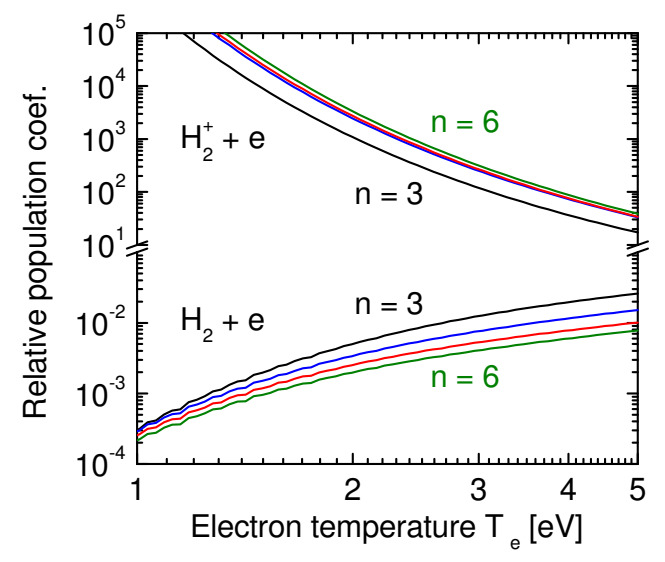

Figure 2. Population coefficients for excitation of the upper Balmer states by $\mathrm{DE}$ and DR reactions relative to the EE channel. The coefficients are calculated with the collisional radiative model Yacora $\mathrm{H}[5]$ for $n_{\mathrm{e}}=3 \times 10^{16} \mathrm{~m}^{-3}$.

state of $\mathrm{H}_{2}$ or via a dissociative auto-ionizing Rydberg state [1]. The threshold energy for this process depends on the initial vibrational state of the $\mathrm{H}_{2}^{+}$molecule and the final electronic state of the excited atom. These threshold energies may also be negative (up to $-1.64 \mathrm{eV}$ [1]) and thus the energy release from the process itself can already easily result in energetic excited atoms. If the radiative decay from the excited states is again faster than thermalizing collisions, these energetic atoms are observable in emission.

Furthermore, due to the fact, that this process is an electron capture process, the total electron kinetic energy is transferred to the potential energy of the intermediate molecular state. Hence, for fixed initial electron energy, the starting level in terms of energy is equal for all the possible succeeding dissociation processes. Atoms in the final state $n=3$ will thus gain more translational energy leaving the molecular potential than atoms in the state $n=6$. Consequently, the $\mathrm{H}_{\alpha}$ line is broadened more than the $\mathrm{H}_{\delta}$ line for the same electron energy.

Moreover, in contrast to the DE process, the DR process is a "top-down-process", since the initial ionic state lies above the final neutral states. Hence, the population coefficients increase with increasing quantum number, which is illustrated in the top part of figure 2. Consequently, the share to a composed line profile should increase with quantum number.

Furthermore, the Balmer lines are each composed of several fine structure (FS) lines, which mostly overlap due to Doppler broadening already at room temperature and thus lead to an additional apparent broadening $\oint$ of the lines. For the $\mathrm{H}_{\alpha}$ line the seven FS lines, for instance, are distributed over about $20 \mathrm{pm}$, while for $\mathrm{H}_{\delta}$ the corresponding range is $6 \mathrm{pm}$ (again seven lines).

In experiment the emission lines are mostly recorded with a spectrometer that has a finite spectral resolution. The measured line profile is thus a convolution of the actual profile with the apparatus profile of the spectrometer. Consequently, the apparatus profile has to be chosen sufficiently narrower than the line profile component of interest. Typical FWHM of applied spectrometers lie in the range of $20-40 \mathrm{pm}$, which gives access only to high energy components. If a low-temperature component is of interest, enhanced spectroscopic systems or other diagnostics like laser diagnostics have to be used.

\section{Experimental setup}

The measurements were taken in the expanding region of a low pressure hydrogen plasma. The experiment vessel is depicted in figure 3 on the left. The electron cyclotron resonance (ECR) plasma HOMER [31] consists of a cylindrical stainless steel vessel of $15 \mathrm{~cm}$ diameter and two magnetic field coils surrounding the vessel. The microwave $(2.45 \mathrm{GHz}, 1 \mathrm{~kW} \max$.$) is$ injected from the top and the heating zone (ECR condition fulfilled at $87.5 \mathrm{mT}$ ) is located just below the first field coil. The prolonged expanding part to the bottom gives a total height of $41 \mathrm{~cm}$. Two lines of sight (LOS) are used for optical emission spectroscopy (OES) in the upper, i. e. heated part of the plasma (LOS I) as well as in the lower, i. e. expanding part (LOS II). Ports perpendicular to the drawing plane at the height of the two LOS are used for Langmuir probe measurements at the cylinder's axis. Typical plasma operation is performed at $0.3 \mathrm{~Pa}$ of hydrogen pressure and $300 \mathrm{~W}$ microwave power.

The spectroscopic system used for OES measurements consists of an Acton SpectraPro spectrometer with a focal length of $0.75 \mathrm{~m}$ and a grating with 1800 lines per mm. It is connected via a $400 \mu \mathrm{m}$ fiber to a lens tube, which is alined to the center of the discharge. The entrance slit of the spectrometer is set to $30 \mu \mathrm{m}$ as a trade-off between light throughput and spectral resolution and the spectrometer is equipped with a CCD camera as detector $(2500 \times 600$ pixels, $\left.12 \times 26 \mu \mathrm{m}^{2}\right)$. The apparatus profile was thoroughly determined by using several different low pressure discharge lamps with rare gases or mercury. Throughout the wavelength range of the spectrometer its apparatus profile resembles a Gaussian profile (digitized by around 10 pixels per peak) with a FWHM of around

$\S$ In the above mentioned widths the FS splitting was neglected, i. e. the given values are valid for a single FS line only. 
$24 \mathrm{pm}$ at $400 \mathrm{~nm}$ down to $21 \mathrm{pm}$ at $650 \mathrm{~nm}$. Absolute calibration is performed by using an Ulbricht sphere as secondary radiation standard and spectra are obtained in units of $\left[\left(\mathrm{m}^{3} \mathrm{~s} \mathrm{~nm}\right)^{-1}\right]$. The emissivity of a line is thus determined by the area under the line profile.

The width $\Delta \lambda_{\text {meas }}$ of a measured line profile is determined by the width $\Delta \lambda_{\text {line }}$ of the actual line profile (including its FS splitting) and the FWHM $\Delta \lambda_{\text {app }}$ of the apparatus profile. Measurement reproducibility and fitting accuracy limit the determination of $\Delta \lambda_{\text {meas }}$ as well as $\Delta \lambda_{\text {app }}$ to about $\pm 1 \mathrm{pm}$. This means that for the present setup, an influence of the temperature of the emitting ensemble on the measured line profile can only be considered significant if it increases $\Delta \lambda_{\text {meas }}$ by at least $2 \mathrm{pm}$ compared to the case with zero temperature. Temperatures of at least $750 \mathrm{~K}$ are thus required for $\mathrm{H}_{\alpha}\left(1300 \mathrm{~K}\right.$ for $\left.\mathrm{H}_{\delta}\right)$ if the FS splitting is taken into account. Apart from that, accurate correlations of the measured widths with the actual temperature can certainly only be assessed for temperatures well above this limit.

\section{Measurements}

\subsection{Relative contributions of excitation channels}

Plasma parameters for a hydrogen plasma at $0.3 \mathrm{~Pa}$ and $300 \mathrm{~W}$ are given in table 1 for the two lines of sight. The electron density is taken from the ion density measured by Langmuir probe while the other parameters are derived via OES. $n_{\mathrm{H}}$ is thereby evaluated from the atom-to-molecule density ratio $\mathrm{H} / \mathrm{H}_{2}$, which in turn can be calculated in different ways: a rather simple line ratio method [11] or via $\mathrm{CR}$ modeling for $\mathrm{H}$ and $\mathrm{H}_{2}$. For both cases the total emissivity of the molecular Fulcher system is required. The most intense lines in this system are the rotational $\mathrm{Q}$ lines $(\Delta \mathrm{J}=0)$ of the diagonal transitions $(\Delta \nu=0)$. Those are measured and scaled to the total system using scaling factors calculated by a ro-vibrationally resolved corona model $[5,32]$.

For the line ratio method of the $\mathrm{H} / \mathrm{H}_{2}$ evaluation, the effective emission rate coefficients for the $\mathrm{H}_{\gamma}$ line and the molecular Fulcher transition are calculated with the $\mathrm{CR}$ model Yacora $\mathrm{H}_{2}$ [5] and a simplified version of the $\mathrm{CR}$ model Yacora $\mathrm{H}$; simplified in the sense that only the EE channel is included here. Like this, the emissivity of the Balmer line only depends on $n_{\mathrm{e}}, T_{\mathrm{e}}$ and $n_{\mathrm{H}}$ and any dependency from other species densities is omitted (as those are usually unknown). The ratio of the effective emission rate coefficients for $\mathrm{H}_{\gamma}$ and Fulcher is thus solely dependent on the atom-to-molecule density ratio and is moreover almost constant for $T_{\mathrm{e}} \approx 1-5 \mathrm{eV}$ and $n_{\mathrm{e}} \approx 10^{16}-10^{17} \mathrm{~m}^{-3}$ [11]. This makes it a very convenient tool for a determination of $n_{\mathrm{H}}$ from spectroscopic measurements.
Table 1. Plasma parameters determined by OES and Langmuir probe at the two positions shown in figure 3 for a hydrogen plasma at $0.3 \mathrm{~Pa}$ and $300 \mathrm{~W}$.

\begin{tabular}{ccccc}
\hline & $n_{\mathrm{e}}\left[\mathrm{m}^{-3}\right]$ & $T_{\mathrm{e}}[\mathrm{eV}]$ & \multicolumn{2}{c}{$n_{\mathrm{H}}\left[\mathrm{m}^{-3}\right]$} \\
\cline { 3 - 5 } & & $\mathrm{H}_{\gamma} /$ Fulcher & full CR \\
\hline LOS I & $4 \times 10^{16}$ & 4.1 & $5.9 \times 10^{18}$ & $5.3 \times 10^{18}$ \\
LOS II & $1 \times 10^{16}$ & 3.6 & $2.2 \times 10^{18}$ & $1.3 \times 10^{18}$ \\
\hline
\end{tabular}

However, the assumption that $\mathrm{EE}$ is the only - or at least the dominant - excitation channel for $\mathrm{H}_{\gamma}$ has to be checked.

Another possibility is to use $\mathrm{CR}$ modeling for $\mathrm{H}$ and $\mathrm{H}_{2}$ including all the excitation channels. Here, Yacora [5] is applied and the input parameters $n_{\mathrm{H}}$, $n_{\mathrm{H}_{2}}, n_{\mathrm{H}_{2}^{+}}$and $T_{\mathrm{e}}$ are varied for a best fit $( \pm 10 \%)$ to the measured emissivities and ratios of the Balmer lines $\mathrm{H}_{\alpha}$ through $\mathrm{H}_{\delta}$ and the Fulcher system, with the absolute pressure as well as the measured ion density being respected. In this way the values of $T_{\mathrm{e}}$ and $n_{\mathrm{H}}$ "full CR" given in table 1 are evaluated. It can be seen, that the difference in $n_{\mathrm{H}}$ between the simple line ratio method and the full evaluation using the $\mathrm{CR}$ models is only negligible for LOS I (smaller than the measurement accuracy of about $\pm 20 \%$ ) but is around $70 \%$ for LOS II, which will be discussed below. Apart from that, the temperature and the densities are lower in the expanding part of the discharge.

Using the CR model Yacora H, furthermore the contributions of the specific excitation channels to the total excitation rate for each Balmer line is determined. The result is shown to the right of figure 3. Any other channel described in Wünderlich and Fantz [5], like for instance mutual neutralization of negative and positive hydrogen ions, is negligible under the present conditions.

It can be seen that in the heated plasma region (LOS I), excitation from the hydrogen atom is by far the dominant channel for all the Balmer lines. All other channels have globally less than $13 \%$ contribution, while the DR channel can even be neglected in the evaluation procedure without affecting the accuracy (illustrated by the error bar of the DR column, which extends until zero). In the expanding region (LOS II) however, excitation by dissociative recombination via $\mathrm{H}_{2}^{+}$shows significant relevance and increasing contribution going from $\mathrm{H}_{\alpha}$ to $\mathrm{H}_{\delta}$ : The $n=6$ level $\left(\mathrm{H}_{\delta}\right)$ is thus populated to $23-40 \%$ by DR. This is mainly attributed to the decreased atomic density and the correspondingly higher share of the DE and DR channels. The slightly decreased electron temperature further shifts the share towards the DR channel.

The differing relevance of the DR channel also reflects in the difference of the $n_{\mathrm{H}}$ determination 

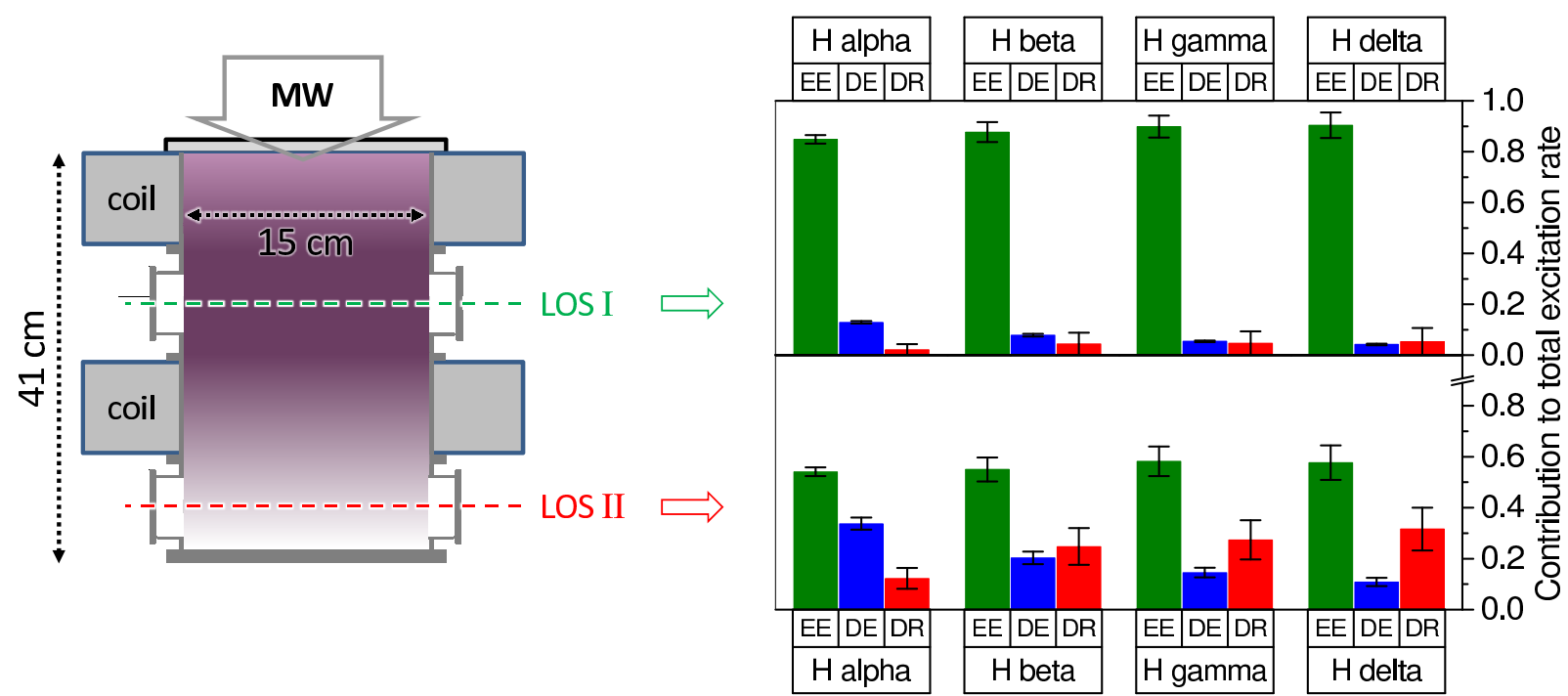

Figure 3. Evaluated contributions of the relevant excitation channels to the total Balmer emission measured at two different plasma regimes at the laboratory experiment HOMER at $0.3 \mathrm{~Pa}$ and $300 \mathrm{~W}$.

methods in table 1. As long as EE is the dominant excitation path for $\mathrm{H}_{\gamma}$, the simple line ratio method holds (LOS I). But as soon as other channels are involved (LOS II), the density via this method is overestimated. In the presented example, DE and DR are responsible for about $42 \%$ of the total excitation to $n=5$, which would be ascribed to the EE channel and thus to the atomic density using the line ratio method, where DE and DR are neglected. $n_{\mathrm{H}}$ is consequently overestimated by around $70 \%$.

\subsection{Line profiles}

Line profiles of the $\mathrm{H}_{\gamma}$ line for the conditions presented above are shown exemplarily in figure 4 (a). The measured spectrum from the mercury line at $435.83 \mathrm{~nm}$ is depicted as well (shifted by $1.78 \mathrm{~nm}$ ) to give the comparison to the apparatus profile. The measured profiles are analyzed by fitting different peak functions, comparable to the approaches described in $[15,17-19,25,27]$. OriginPro (OriginLab Corporation, Northampton, MA, USA, Version 2019b) is used for this purpose with its included fitting routines. Single peak functions (Gaussian, Lorentzian) are fitted with four free parameters (offset, center, FWHM, area) until convergence is reached. For the double Gaussian function (see below), the parameters 'offset' and 'center' are shared for both Gaussian parts, while the two widths and the two areas are fitted independently leading to six free parameters (offset, center, FWHM1, area1, FWHM2, area2). Results from different peak functions $\|$ are rated by eye in view of the best reproduction of the measured profile, especially in the wings.

In figure 4 (a) it can be seen that the measured profile at LOS I is very close to the apparatus profile and therefore, any kind of broadening mechanisms cannot be resolved. A (single) Gaussian peak fit yields very good agreement and a FWHM of $26 \mathrm{pm}$. For the expanding region at LOS II, however, the measured profile is clearly broader than the apparatus profile and a Gaussian peak fit would give a width of around $30 \mathrm{pm}$. However, considerable deviations between the fit and the measured profile emerge, as is illustrated in figure 4 (b), where fits with different peak functions are shown. The apparatus profile is plotted again for comparison. The single Gaussian function (green line) shows the deviation from the measured profile especially in the wings of the profile. This observation is true for all the Balmer lines at LOS II and indicates that in the expanding region the line profile for the Balmer lines is not only determined by the apparatus profile of the spectrometer but consists of more than one component.

Since the deviation from the apparatus profileor, conterminously, from the Gaussian peak fit-is worse in the wings of the peak, a Lorentzian peak fit or a double-peak-fit are the logical next choices, shown in figure 4 (b). While the Lorentzian profile (blue line) clearly overestimates the evolution in the wings,

|| In the following, where not particularly specified, 'Gaussian' (and 'Lorentzian') peak fits are meant as single peak function. The 'double Gaussian' peak fit is specified at each occurrence. 


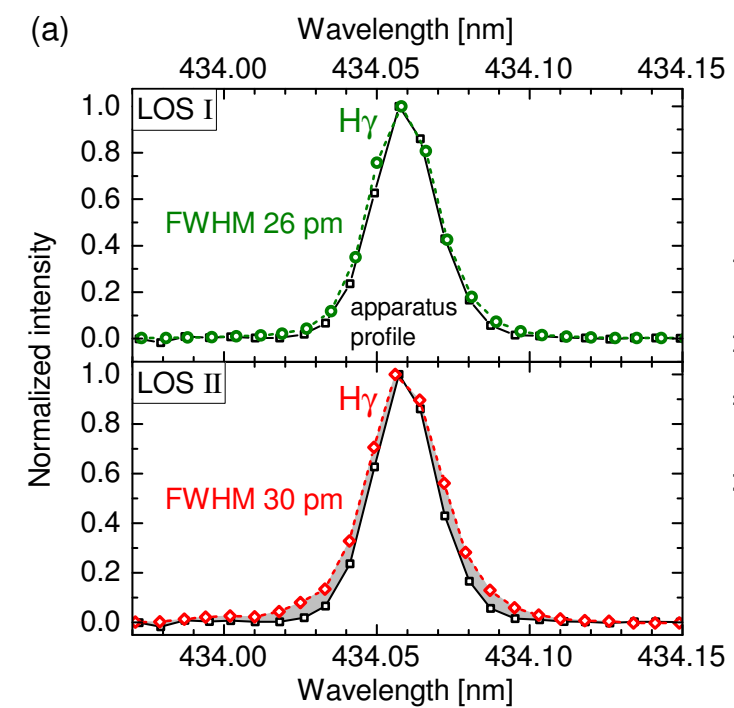

(b)

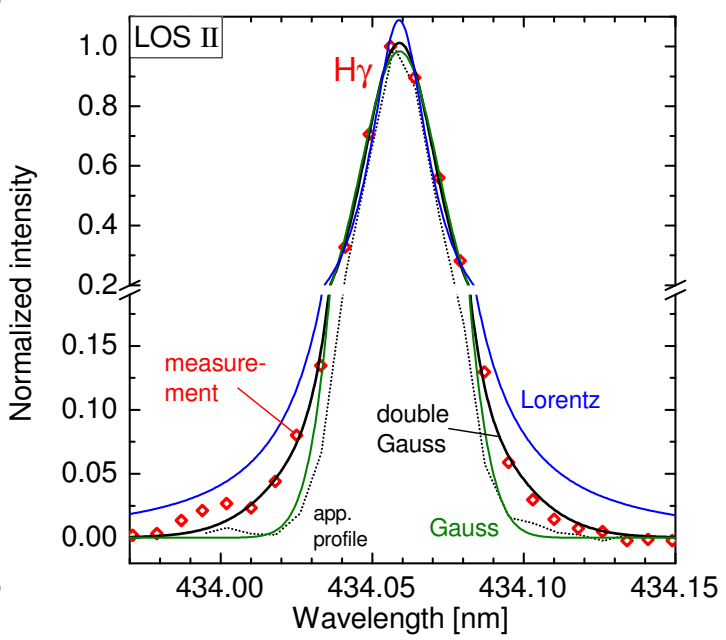

Figure 4. (a) Line profiles of the $\mathrm{H}_{\gamma}$ line measured at the two lines of sight and compared to the apparatus profile of the spectrometer (shifted mercury line in black). (b) $\mathrm{H}_{\gamma}$ line measured at LOS II in comparison with three different peak fits (Gauss, Lorentz, double Gauss) and the measured apparatus profile (dotted line).

applying the sum of two Gaussian profiles at the same central wavelength gives very good agreement with the measured line profile. The two distinct contributions of the combined double Gaussian peak fit are shown in figure 5 (a): a broad component with a FWHM of $60 \mathrm{pm}$ and an area share (= area1/(area1+area2)) of $32 \%$ emerges next to a narrow component with a width of $25 \mathrm{pm}$ and a share of $68 \%$. The narrow component furthermore agrees very well with the apparatus profile and its FWHM agrees well with the one measured at LOS I if the uncertainty of $\pm 1 \mathrm{pm}$ is taken into account.

The double peak fitting can be applied to all the Balmer lines and the results are shown in figure 5 (b). The FWHM of the narrow and the broad component are depicted together with the respective area share of the broad part. Furthermore, the corresponding width of the apparatus profile is given and the gray shaded area illustrates the fine structure splitting as described to the end of section 3. Additionally, the FWHM of the Gaussian peak fits for the line profiles measured at LOS I are given (green diamonds) and the FWHM of the $\mathrm{Q}$ lines of the $\mathrm{H}_{2}$ Fulcher system is shown (orange circle). For all the Balmer line profiles at LOS II, the narrow component agrees very well with the one measured at LOS I. Furthermore, it can be seen that for the individual Balmer transitions this profile component is already determined by the convolution of the apparatus profile with the distributed fine structure lines. The $\mathrm{Q}$ lines of the Fulcher system do not show such a distribution of lines and thus, their FWHM for both LOS agree very well with the corresponding apparatus profile (which is virtually the same as for the $\mathrm{H}_{\alpha}$ line).

Hence, taking account of the further considerations in section 3 , it can be stated that the narrow component of the Balmer lines in the expanding region of the plasma (as well as the profile in the heated region) most certainly emerges from electron impact excitation from "cold" atoms (equation (1)). The resolution of the spectroscopic system is not sufficient to give an accurate statement about the actual temperature $T_{\mathrm{H}}$, but the upper limit is already described in section 4 , hence $T_{\mathrm{H}} \leq 750 \mathrm{~K}$. The lower limit can be defined by the gas temperature, i. e. the temperature of the hydrogen molecules, which is evaluated using the rotational lines of the Fulcher system [33]. $T_{\mathrm{H}_{2}}$ is thus determined to be around $450 \mathrm{~K}$ in the expanding region (480 K at LOS I), which agrees well with the fact, that no broadening beyond the apparatus profile could be detected for the rotational lines. Consequently, the temperature of the cold atoms, responsible for the narrow component of the Balmer line profiles, can be estimated to be in the range $450-750 \mathrm{~K}$.

The broad component of the Balmer line profiles in the expanding region is, however, much wider than the apparatus profile: The FWHM of this component ranges from $102 \mathrm{pm}$ for $\mathrm{H}_{\alpha}$ to $51 \mathrm{pm}$ for $\mathrm{H}_{\delta}$, while the respective area shares increase from $12 \%$ to more than $35 \%$. Such broad line profiles have to be correlated to energetic excited atoms with energies in the $\mathrm{eV}$ range and their origin is discussed in the next section. 
(a)

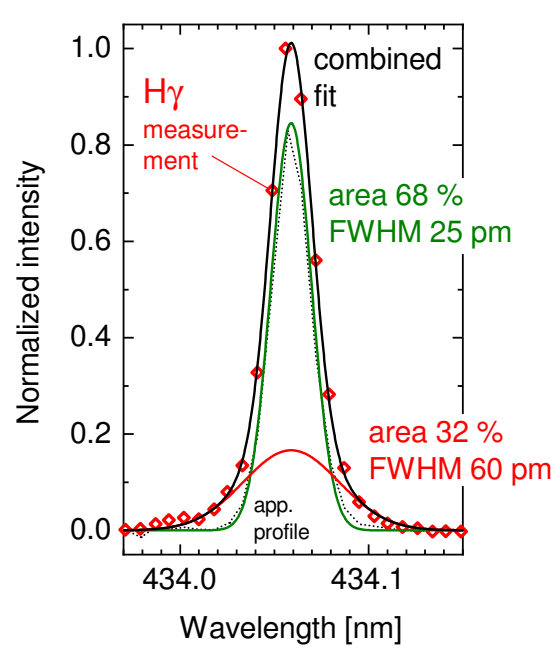

(b)

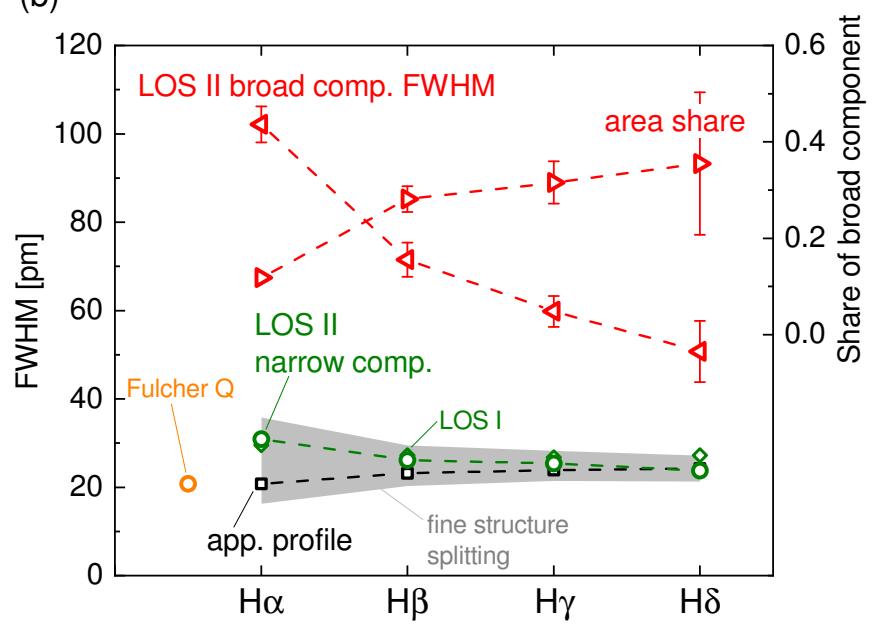

Figure 5. (a) Double Gaussian peak fit of the $\mathrm{H}_{\gamma}$ line measured at LOS II. The measured apparatus profile is again depicted as dotted line, normalized to the peak value of the narrow component. (b) FWHM of the narrow (green circles) and broad component (red triangles, left axis) as well as area share of the broad part (red triangles, right axis) for the Balmer lines $\mathrm{H}_{\alpha}$ through $\mathrm{H}_{\delta}$ at LOS II. For comparison the respective apparatus profile (black squares), the FWHM of the line profiles at LOS I (green diamonds), the FWHM of the Fulcher Q lines at LOS I \& II (orange circle) and the fine structure splitting (grey shaded area) are depicted.

\section{Discussion and implications of the broad component}

As described in section 3, several processes can lead to energetic excited atoms with translational energies in the range of some $\mathrm{eV}$, namely excitation of energetic ground state atoms (produced by the dissociation process) through the EE channel (1), direct dissociative excitation of hydrogen molecules (DE (2)) and dissociative recombination of $\mathrm{H}_{2}^{+}$ions (DR (3)). While each channel leads to decreasing line widths with increasing quantum number (in agreement with the line profile analysis in figure $5(\mathrm{~b})$ ), they differ in their expected relative share to the total line profile among the Balmer lines: for the EE channel, the share of the energetic ensemble should be constant for all the lines, whereas for the DE channel it should decrease going from $\mathrm{H}_{\alpha}$ to $\mathrm{H}_{\delta}$. The reason is the expected relative population contribution of the channels for each Balmer line; for DE this is confirmed by the $\mathrm{CR}$ analysis of the measured emissivities illustrated in figure 3: for LOS II the blue bars show a decreasing relevance of DE for increasing quantum number. As those two mechanisms are thus in contradiction to the measured evolution of the share of the broad component of the Balmer line profiles, DE as well as EE can be ruled out as being responsible for the broad line profile component. A "top-down-process" like the DR channel, however, increases its share from $\mathrm{H}_{\alpha}$ to $\mathrm{H}_{\delta}$, which is confirmed by the red bars at LOS II in figure 3 . This behavior agrees very well with the analysis of the broad component.
The indicated correlation is emphasized in figure 6 , where the share of the broad component (black line) is depicted together with the evaluated excitation contribution from dissociative recombination (red shaded area) and-for comparison-from dissociative excitation (blue shaded area). The agreement of the DR share with the determined share of the broad component for each of the Balmer lines is excellent. Hence, the observed behavior of the broad component of the line profiles of the Balmer series is traced back to the DR process for exciting the upper Balmer states: increasing share at the line profile from $\mathrm{H}_{\alpha}$ to $\mathrm{H}_{\delta}$ due to increasing population contribution via $\mathrm{DR}$ and decreasing line width from $\mathrm{H}_{\alpha}$ to $\mathrm{H}_{\delta}$ due to decreasing energy release during the DR process.

This observation is true for a wide pressure range, which is demonstrated in figure 7 for measurements between 0.3 and $3 \mathrm{~Pa}$. For this purpose a meshed grid was introduced just below the first field coil of the ECR plasma. In this configuration the heating zone is slightly shifted upwards and the expanding region of the plasma is already located at LOS I. The setup in this configuration is described in detail in Kurutz et al. [31]. At $0.3 \mathrm{~Pa}$ the plasma conditions for this configuration are practically identical to the conditions at LOS II without the meshed grid, which was described above. Part (a) of figure 7 depicts in the bottom part the corresponding electron temperatures and atomic densities, confirming comparable values at $0.3 \mathrm{~Pa}$ to those given in table 1 , LOS II. The parameters are evaluated using the full $\mathrm{CR}$ models for $\mathrm{H}$ and $\mathrm{H}_{2}$ as described in section 5.1. Furthermore, over the entire 


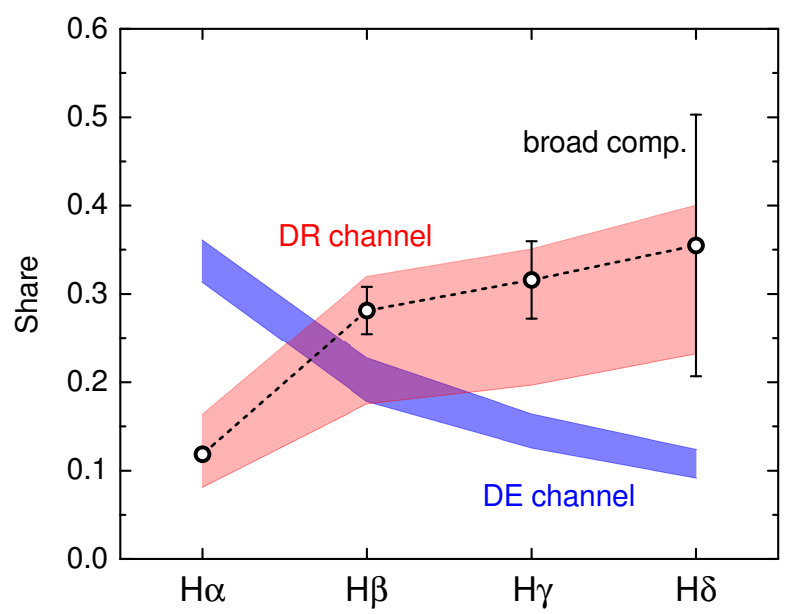

Figure 6. Measured area share of the broad component of the Balmer line profiles (black line, taken from figure 5 (b)) in comparison with the evaluated contributions of dissociative excitation (DE, blue shaded area) and dissociative recombination (DR, red shaded area) to the total emissivity of the particular Balmer lines via the CR model Yacora $\mathrm{H}$ (taken from figure 3, LOS II).

pressure range all the Balmer lines show a broadened line profile, which is nicely fitted by a double Gaussian peak fit.

In the top part of figure 7 (a) the evaluated shares of the broad component of the $\mathrm{H}_{\gamma}$ line are shown together with the respective evaluated contributions of dissociative recombination via the CR model. The decreasing electron temperature with increasing pressure shifts the excitation equilibrium further towards the DR channel, while the increasing atomic density acts in the opposite direction (towards the EE channel). The result is a nearly constant contribution of DR with increasing pressure, which agrees well with the virtually constant area share of the broad component of the line profile. Part (b) of the figure shows this analysis for all the Balmer lines at three different pressures: for all the pressures and for all the Balmer lines the contribution of DR clearly agrees well with the share of the broad component of the line profile.

Since it is shown that DR is responsible for the broad component of the Balmer line profile, the information can be used for spectroscopic diagnostics. More specifically, the simple line ratio method for determining the atomic density via OES in LPLT hydrogen plasmas can be enhanced. Figure 8 shows the evaluation of the atomic density for the pressure scan via three different methods. (A) and (B) are already described in section 5.1 and relate to the simple line ratio method [11] and the full evaluation via the CR models Yacora $\mathrm{H}$ and $\mathrm{H}_{2}$ [5], respectively. The values from the latter evaluation are also shown in figure 7 (a). Neglecting the population channels besides EE in the evaluation (method (A)) consequently leads to an overestimation of $n_{\mathrm{H}}$ of up to more than a factor of two (at $3 \mathrm{~Pa}$ ).

For method $(\mathrm{C})$ it is considered that the broad component of the Balmer line profiles arises from DR and is thus not correlated to the atomic density, which in turn should only be responsible for the narrow component, as described in section 5.2. Consequently, using only the narrow component for an evaluation of $n_{\mathrm{H}}$ via the line ratio method $\mathrm{H}_{\gamma} /$ Fulcher, the agreement with the full $\mathrm{CR}$ evaluation is excellent. Only at high pressure a deviation between methods (B) and (C) emerges. Here, the contribution of $\mathrm{DE}$ is getting relevant, which is still neglected with method (C) since it cannot be assessed via the line profile. Thus, a slight overestimation still occurs at high pressure. This demonstrates that the simple line ratio method together with an analysis of the Balmer line profile can be used to determine the atomic density with high accuracy, even without the full evaluation of the Balmer series and the Fulcher emission via CR models. The prerequisite is an observable broad component of the line profile and the confirmation that this component can be related to processes aside from EE.

\section{Conclusions}

In low pressure low temperature hydrogen plasmas electron impact excitation, dissociative excitation and dissociative recombination are prominent excitation channels for the Balmer series. Furthermore, these processes can have a specific influence on the translational energy of excited atoms which is correlated to the width of the respective line profiles. The expanding region of a LPLT hydrogen plasma was analyzed in this regard spectroscopically: the combination of evaluating the absolute emissivities of the first four Balmer lines $\left(\mathrm{H}_{\alpha}-\mathrm{H}_{\delta}\right)$ by $\mathrm{CR}$ modeling and analyzing the respective line profiles, revealed that the DR channel has a significant contribution to the excitation of the Balmer series and that it gives rise to a broad component in the line profile of all the Balmer lines.

This paper thus confirms the well-known fact, that a thorough analysis of the Balmer line profiles can reveal excitation channels besides electron impact excitation. Furthermore, it emphasizes, that simple line ratio methods may lose their validity if such processes are neglected. Using the line profile analysis it is, however, illustrated, that the validity range of the line ratio technique to determine the atomic hydrogen density $\left(\mathrm{H}_{\gamma} /\right.$ Fulcher $)$ can be extended to the class of expanding hydrogen plasmas, for which formerly full collisional-radiative modeling of the Balmer series 


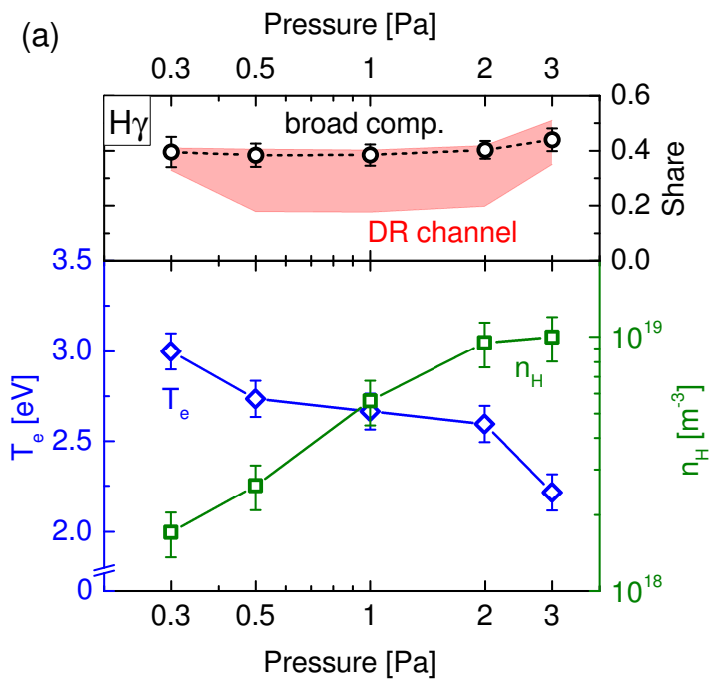

(b)

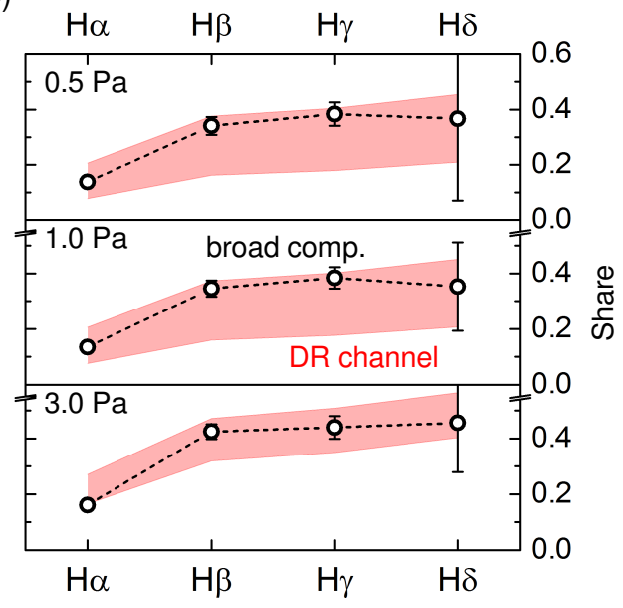

Figure 7. (a) Electron temperature and atomic hydrogen density for varying pressure (at $300 \mathrm{~W}$ ) at LOS I with introduced meshed grid (see [31]) and corresponding shares of the broad component of the line profile of the $\mathrm{H}_{\gamma}$ line (black line) together with the contribution of the dissociative recombination channel (DR) to the total emission (red shaded area). (b) Shares of the broad line component and the DR channel for the measured Balmer series for selected pressures.

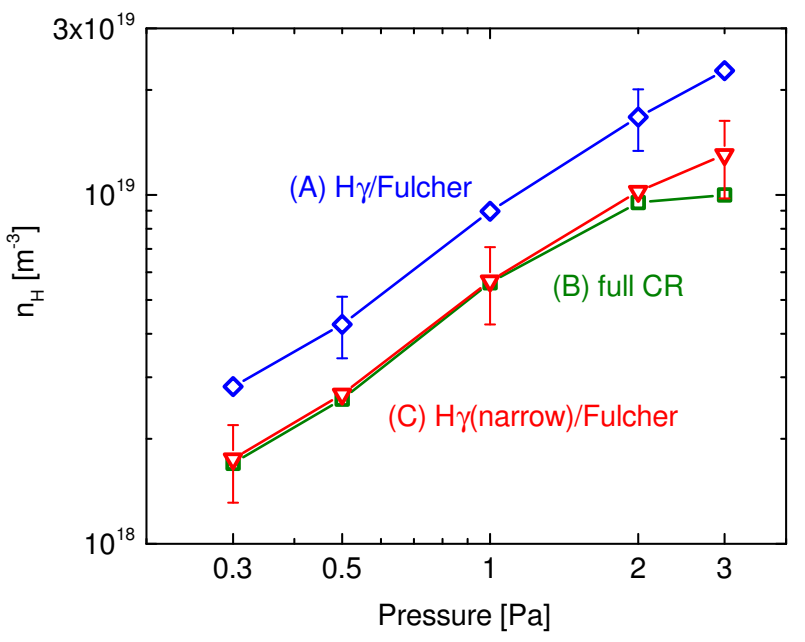

Figure 8. Atomic hydrogen density evaluated by different methods: (A) Line ratio method taking the $\mathrm{H}_{\gamma}$ line and the $\mathrm{H}_{2}$ Fulcher system, (B) evaluation using the full CR models for $\mathrm{H}$ and $\mathrm{H}_{2}$, and (C) line ratio method as in (A), but taking only the narrow component of the $\mathrm{H}_{\gamma}$ line.

as well as the $\mathrm{H}_{2}$ Fulcher emission was necessary. Here, the broad component of the line profiles, which is ascribed to dissociative recombination, is omitted for the line ratio technique and the results virtually coincide with full CR modeling.

\section{Acknowledgments}

The authors thank U. Kurutz, former member of the AG Experimentelle Plasmaphysik (Universität Augsburg) and Max-Planck-Institut für Plasmaphysik (Garching), for performing part of the measurements and moreover for fruitful discussions regarding the results.

\section{References}

[1] Janev R K, Reiter D and Samm U 2003 Collision Processes in Low-Temperature Hydrogen Plasmas Berichte des Forschungszentrums Jülich JUEL-4105 Forschungszentrum Jülich

[2] Fujimoto T, Sawada K and Takahata K 1989 J. Appl. Phys. $662315-2319$

[3] Hassouni K, Gicquel A, Capitelli M and Loureiro J 1999 Plasma Sources Sci. Technol. 8 494-512

[4] Okamoto A, Kado S, Sawada K, Kuwahara Y, Iida Y and Tanaka S 2007 J. Nucl. Mater. 363-365 395-399

[5] Wünderlich D and Fantz U 2016 Atoms 426

[6] Devyatov A M, Kalinin A V and Miiovich S R 1991 Optics and Spectroscopy 71 525-526

[7] Schulz-von der Gathen V and Döbele H F 1996 Plasma Chemistry and Plasma Processing 16 461-486

[8] Kado S, Kajita S, Yamasaki D, Iida Y, Xiao B, Shikama T, Oishi T, Okamoto A and Tanaka S 2005 J. Nucl. Mater. 337-339 166-170

[9] Lavrov B P, Pipa A V and Röpcke J 2006 Plasma Sources Science and Technology 15 135-146

[10] Lavrov B P, Pipa A V and Röpcke J 2006 Plasma Sources Science and Technology 15 147-155

[11] Fantz U, Falter H, Franzen P, Wünderlich D, Berger M, Lorenz A, Kraus W, McNeely P, Riedl R and Speth E 2006 Nucl. Fusion 46 S297-S306

[12] Cappelli A L, Gottscho R A and Miller T A 1985 Plasma Chemistry and Plasma Processing 5317

[13] Pealat M, Taran J P E, Bacal M and Hillion F $1985 \mathrm{~J}$. Chem. Phys. 824943

[14] Baravian G, Chouan Y, Ricard A and Sultan G 1987 J. Appl. Phys. 615249

[15] Vrhovac S B, Radovanov S B, Bzenic S A, Petrovic Z L and Jelenkovic B M 1991 Chem. Phys. 153233

[16] Radovanov S B, Dzierzga K, Roberts J R and Olthoff J K 1995 Applied Physics Letters 662637 
[17] Mills R L, Ray P C, Dhandapani B, Mayo R M and He J 2002 J. Appl. Phys. 927008

[18] Phelps A V 2011 Plasma Sources Sci. Technol. 20043001

[19] Jimenez-Redondo M, Carrasco E, Herrero V J and Tanarro I 2013 Plasma Sources Sci. Technol. 22025022

[20] Lisitsa V S 1977 Sov. Phys. Usp. 20603

[21] Ashkenazy J, Kipper R and Caner M 1991 Phys. Rev. A 43 5568

[22] Gigosos M A and Cardenoso V 1996 J. Phys. B: At. Mol. Opt. Phys. 294795

[23] Wenzel U, Behringer K, Carlson A, Gafert J, Napiontek B and Thoma A 1999 Nuclear Fusion 39 873-882

[24] Potzel S, Dux R, Mller H W, Scarabosio A, Wischmeier $\mathrm{M}$ and ASDEX Upgrade team 2014 Plasma Physics and Controlled Fusion 56025010

[25] Kask N E, Leksina E G, Michurin S V, Fedorov G M and Chopornyak D B 2015 Quantum Electronics 45 527-532

[26] Rosato J, Kieu N, Meireni M, Sheeba R, Koubiti M, Marandet Y, Stamm R, Verhaegh K, Duval B, The TCV team and The MST1 team 2018 Contributions to Plasma Physics 58 578-582

[27] Parigger C G, Helstern C M, Gautam G and Drake K A 2019 Journal of Physics: Conference Series 1289 012001

[28] Freund R S, Schiavone J A and Brader D F 1976 J. Chem. Phys. 641122

[29] Thorne A, Litzén U and Johansson S 1999 Spectrophysics: Principles and Applications (Berlin Heidelberg: Springer-Verlag)

[30] Fubiani G, Garrigues L, Hagelaar G, Kohen N and Boeuf J P 2017 New J. Phys. 19015002

[31] Kurutz U, Friedl R and Fantz U 2017 Plasma Phys. Control. Fusion $\mathbf{5 9} 075008$

[32] Dietrich S 2010 Verifikation von optischen Diagnostikmethoden an $\mathrm{H}_{2} / D_{2}-$ Plasmen Ph.D. thesis University of Augsburg

[33] Briefi S, Rauner D and Fantz U 2017 J. Quant. Spectrosc. Radiat. Transf. 187135 